\title{
Fabrication of Hierarchically Ordered Hybrid Structures over Multiple Length Scales via Direct Etching of Self-Organized Polyhedral Oligomeric Silsesquioxane (POSS) Functionalized Block Copolymer Films
}

\author{
Teruaki HAYAKAwa,,$^{1, \dagger}$ Makoto SEINO, ${ }^{1}$ Raita GoseKI, ${ }^{1}$ \\ Tomoyasu HIRAI, ${ }^{1}$ Ryohei KIKUCHI, ${ }^{1}$ Masa-aki KAKIMOTO, ${ }^{1}$ \\ Masatoshi TOKITA, ${ }^{1}$ Hideaki YOKOYAMA, ${ }^{2}$ and Shin HORIUCHI ${ }^{2}$ \\ ${ }^{1}$ Department of Organic and Polymeric Materials, Tokyo Institute of Technology, \\ 2-12-1-S8-26, O-okayama, Meguro-ku, Tokyo 152-8552, Japan \\ ${ }^{2}$ Nanotechnology Research Institute, National Institute of Advanced Industrial Science and Technology, \\ Tsukuba Central 5, 1-1-1, Higashi, Tsukuba 305-8565, Japan
}

(Received September 26, 2005; Accepted February 9, 2006; Published May 17, 2006)

\begin{abstract}
A novel fabrication of hierarchically ordered organic and inorganic hybrid structures at length scales ranging from nanometers to micrometers was demonstrated by oxygen plasma treatment of self-organized silicon-containing block copolymer films. The rod-coil type silicon-containing block copolymer, polystyrene- $b$-polyisoprene with polyhedral oligomeric silsesquioxane (POSS) -modified side chains, was successfully synthesized by hydrosilation of polystyrene- $b$-poly(1,2-ran-3,4-isoprene) block copolymer with hydrido-heptacyclopentyl substituted POSS. The films were prepared from the polymer solution by casting on silicon wafers under a moist air flow. The self-organized microstructures were investigated by electron microscopy. It was found that the hexagonally packed micropores and phaseseparated nanodomains were formed within the films. Oxygen plasma etching of the films provided novel hierarchically ordered hybrid structures. [doi:10.1295/polymj.PJ2005140]

KEY WORDS Block Copolymer / Polyhedral Oligomeric Silsesquioxanes (POSS) / Rod-Coil / Hierarchically Structure / Hybrid / Nano-dots / Oxygen Plasma /
\end{abstract}

Fabrication of nanometer and micrometer scale ordered structures at low cost is an essential objective of a wide range of current science and technology for the miniaturization of electronic, optic ${ }^{1,2}$ and magnetic devices, ${ }^{3-5}$ and sensors ${ }^{6-9}$ etc. Numerous methods such as photolithography ${ }^{10}$ or chemical vapor deposition ${ }^{11}$ have successfully fabricated a variety of ordered structures over a wide range of length scales, but still require tedious multiple-step processes with high costs. On the other hand, instead of these approaches, combinations of various kind of molecular self-organization and facile direct lithographic techniques are of particular recent interests. ${ }^{12-14}$ The advantage of using the molecular self-organization is that one can directly produce ordered two- or three-dimensional hierarchically structures and tailored surfaces by a single step.

Block copolymers are superior self-organizing materials with tunable built-in structures and tailored properties. ${ }^{15,16}$ Many of coil-coil type block copolymers form a variety of periodic phase-separated structures such as spheres, cylinders, and lamellae with typical dimension of 5-50 nm. The sizes and shapes of the nanostructures can be controlled by manipulating chain length, volume fraction of each block and chemical functionality. Among various block copolymers, silicon-containing block copolymers have been successfully used as a template for direct patterning of ordered nanostructures by oxygen plasma treatment. ${ }^{17-19}$ The silicon-containing segments in the block copolymer offer high etch resistance to oxygen plasma compared to hydrocarbon segments due to the stability of silicon oxide, which is the product of the oxygen plasma etching. Thus, the phase-separated silicon-containing structures selectively remain as nano-patterns after the oxygen plasma treatment. However, the length scales of the direct patterning have been limited to the dimension of the phase-separated nanostructures.

In comparison to the coil-coil type block copolymers, rod-coil type block copolymers containing a variety of stiff rigid-rod like molecules such as mesogenic units or organic semiconducting units offer intriguing possibilities for formation of a wide range of functional self-organized structures at length scales from nanometers to micrometers. ${ }^{20}$ We reported a semi-rod-coil type block copolymer, polystyrene$b$-polyisoprene with oligothiophene-modified side chains (PS- $b$-POTI) and the hierarchically structure within the film which prepared from the polymer solution by casting under a moist air flow. ${ }^{21}$ Three tiers of the ordered empty micropores, the walls of which con-

${ }^{\dagger}$ To whom correspondence should be addressed (Tel: +81-3-5734-2429, Fax: +81-3-5734-2875, E-mail: hayakawa@op.titech.ac.jp). 
sist of the ordered nanostructures of PS- $b$-POTI, are formed in the polymer thin film. Moreover, it was recently revealed that the rod-coil type block copolymers of polystyrene- $b$-oligothiophenes form a chemically heterogeneous surface in the microporous film. ${ }^{22}$ The interiors of the pores on the surface were covered with the rod segment of oligothiophene, whereas the surface of the flat area was covered with the coil segment of polystyrene. The mutual repulsion of the dissimilar blocks and the packing of the rod molecules in the rod-coil type block copolymers lead to the formation of a variety of unique structures which are not seen in the self-assembling of the coil-coil type block copolymers.

Most of siloxane-based materials are viscous liquids at room temperature due to the flexible chains, but some of cubic type polyhedral oligomeric silsesquioxanes (POSS) possesses high crystallinity and precisely nanometer-sized structures. ${ }^{23,24}$ Thus, the cubic type POSS may have a good candidate for the rigid-rod molecules of the rod-coil type silicon-containing block copolymers. Herein, we report a novel fabrication of the hierarchically ordered organic and inorganic hybrid structures at length scales from nanometers to micrometers over an area of more than 1 square centimeter using a rod-coil type silicon-containing block copolymer and direct etching technique under oxygen plasma. We examined to synthesize the new rod-coil silicon-containing block copolymer, polystyrene- $b$-polyisoprene modified with heptacyclopentyl substituted POSS side chain (PS- $b$-PIPOSS), and prepare the self-organized films from the solution by casting under a moist air flow. The resulting films were etched under oxygen plasma and fabricated the hierarchically ordered hybrid structures.

\section{EXPERIMENTAL}

\section{Materials}

Hydrido-heptacyclopentyl substituted polyhedral oligomeric silsesquioxane (Hydrido-heptacyclopentyl substituted POSS) was purchased from Aldrich. Unless otherwise noted, all reagents were purchased from Aldrich, Tokyo Kasei Kogyo Co. (TCI), and Wako Pure Chemical Industries (WAKO), and used without further purification. Tetrahydrofuran (THF) was distilled over sodium/benzophenone. Toluene was distilled over $\mathrm{CaH}_{2}$. Styrene was purified by washing with $\mathrm{NaOH}$ aq $(1 \mathrm{M})$ to remove inhibitor before stirring with $\mathrm{CaH}_{2}$ for $24 \mathrm{~h}$, then vacuum-distilled. Isoprene was distilled over $\mathrm{CaH}_{2}$ after stirring for $24 \mathrm{~h}$. First distilled styrene and isoprene were stirred with dibutylmagnesium for $6 \mathrm{~h}$, and then finally vacuumdistilled into a cold trap of a monomer reservoir. Silicon wafers were used as received.

\section{Measurement}

Infrared spectrum was recorded on a JASCO FT/IR460 Plus spectrometer. ${ }^{1} \mathrm{H}$ and ${ }^{13} \mathrm{C}$ NMR spectra of the polymer solutions in deuterated chloroform with tetramethylsilane as an internal standard were obtained with a JEOL JNM-AL 300 spectrometer at $300 \mathrm{MHz}$ and $75 \mathrm{MHz}$, respectively. Gel permeation chromatography (GPC) measurements were carried out using a JASCO HBPX $880 \mathrm{Pu}$, two polystyrene-divinylbenzene columns (Shodex GPC803L and Shodex GPC804L), and a Shodex RI-71 refractive index detector. THF was used as an eluent with $1.0 \mathrm{~mL} \mathrm{~min}^{-1}$ flow rate at $40^{\circ} \mathrm{C}$. Molecular weights are quoted with monodisperse polystyrenes as standards. Thermal analyses of the copolymers, thermogravimetric analysis (TGA) and differential scanning calorimatry (DSC), were carried out using a Seiko SSC/6000 (TG/DTA 6200 and DSC6200) thermal analyzer with $c a .5 \mathrm{mg}$ of samples at a heating rate of $10^{\circ} \mathrm{Cmin}^{-1}$. Nitrogen was used as purge gas at a flow rate of $50 \mathrm{~mL} \mathrm{~min}^{-1}$ in TGA and DSC measurements. A polarizing microscope (Olympus BH2) equipped with a high-speed camera (Photoron DVR) was used to observe self-assembling behavior of micrometer-sized water droplets during a formation process of films. Scanning electron microscope (SEM) images were obtained using a HITACHI FE-SEM S-800 equipped with a field emission gun (FESEM). A leo922 Energy-filtering transmission electron microscope (EFTEM), with which an omega-type electron spectrometer is equipped, was operated at an acceleration voltage of $200 \mathrm{kV}$ to observe the cross-sections of the films. For specimen preparation, the polymer solution was cast on a cured epoxy plate (Queto812), and then it was embedded in the same epoxy resin to be cured at $60^{\circ} \mathrm{C}$ overnight. 50 -nm-thick sections of the films were cut by ultramicrotomy. Energy-filtered images were acquired by a $2 \mathrm{~K} \times 2 \mathrm{~K}$ slow scan CCD camera. Atomic Force Microscopy (AFM) measurements were performed on a Seiko model SPA 300. Small-angle X-ray scattering (SAXS) profiles are obtained using a Bruker NanoSTAR $(45 \mathrm{kV} / 110 \mathrm{~mA})$ with a 2D-PSPC detector (camera length $1055 \mathrm{~mm}$ ). The etching process to expose the hierarchically structures to the surface was performed by reactive ion etcher (SAMCO Compact Etcher FA-1) with an oxygen flow rate of $3 \mathrm{~mL} \mathrm{~min}^{-1}$, a pressure of $8.0 \mathrm{~Pa}$, and a power density of $50 \mathrm{~W} \mathrm{~cm}^{-2}$.

\section{Polystyrene-b-poly(1,2-isoprene-ran-3,4-isoprene) (PS- $b-P I)$}

Polystyrene- $b$-poly (1,2-ran-3,4-isoprene) block copolymer, namely, $\mathrm{PS}_{1200}-b$ - $\mathrm{PI}_{94}$, (the subscripts indicate the number of repeat monomer units) was synthesized by anionic polymerization, as reported previously. ${ }^{25}$ 

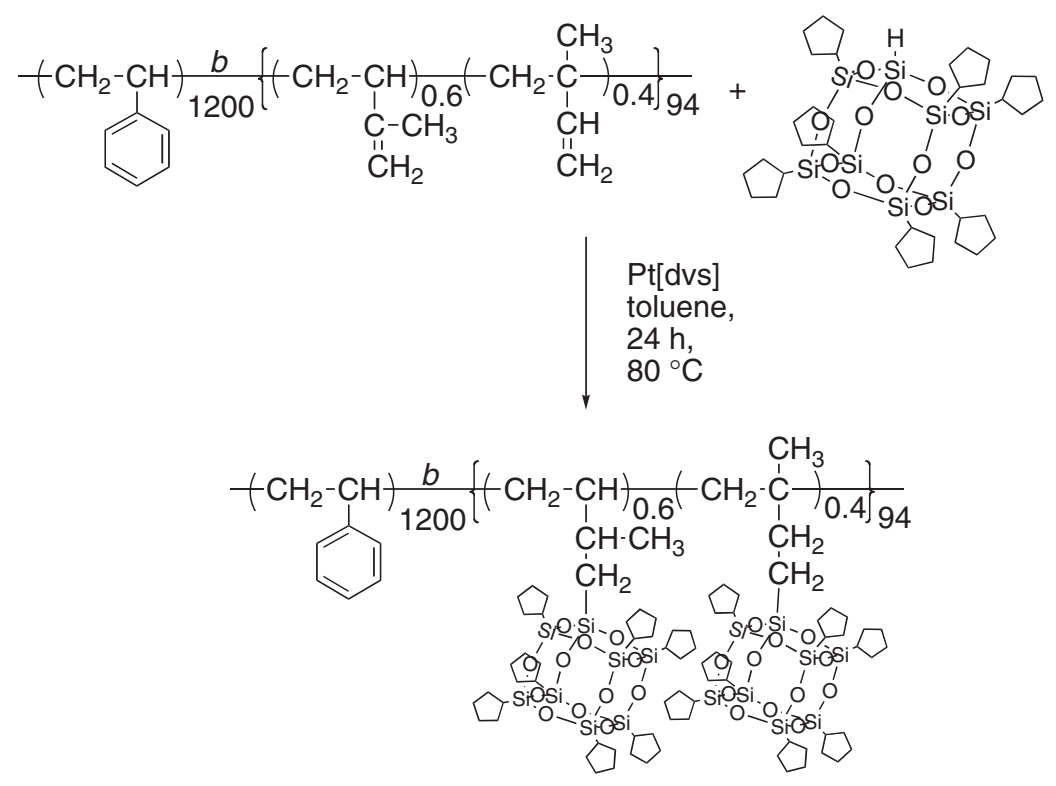

Scheme 1.

The number-average of molecular weight $\left(M_{\mathrm{n}}\right)$ of the polystyrene precursor of $\mathrm{PS}_{1200}-b$ - $\mathrm{PI}_{94}$ is 125,000 in GPC measurement. ${ }^{1} \mathrm{H}$ NMR spectroscopy was used to determine the composition of the block copolymers. The PS- $b$-PI had about 40\% 1,2-addition and $60 \%$ 3,4-addition of isoprene units in the PI block. The molecular weight of the PI block of $\mathrm{PS}_{1200}-b-\mathrm{PI}_{94}$ was 6,500, which was calculated from the compositions determined by ${ }^{1} \mathrm{H}$ NMR in $\mathrm{CDCl}_{3}$. GPC chromatogram of $\mathrm{PS}_{1200}-b-\mathrm{PI}_{94}$ gives $M_{\mathrm{n}}$ of 130,000 $\left(M_{\mathrm{w}} / M_{\mathrm{n}}: 1.12\right) . \mathrm{IR}(\mathrm{NaCl}): v\left(\mathrm{~cm}^{-1}\right)=3060,3030$, 2925, 2855 (C-H, st), 1635, $910(\mathrm{C}=\mathrm{C}, \mathrm{st}), 1600$ (benzene, st). ${ }^{1} \mathrm{H} \mathrm{NMR}\left(300 \mathrm{MHz}, \mathrm{CDCl}_{3}\right) \delta(\mathrm{ppm})=$ 0.90-2.31 (br, $\left.\mathrm{CH}_{3}, \mathrm{CH}_{2}, \mathrm{CH}\right), 4.63$ (br, $-\mathrm{C}\left(\mathrm{CH}_{3}\right)=$ $\left.\mathrm{CH}_{2}, 2 \mathrm{H}\right), 4.86\left(\mathrm{br},-\mathrm{CH}=\mathrm{CH}_{2}, 1 \mathrm{H}\right), 5.72(\mathrm{br},-\mathrm{CH}=$ $\left.\mathrm{CH}_{2}, 1 \mathrm{H}\right), 6.38-7.24$ (br, benzene, $5 \mathrm{H}$ ).

Polystyrene-b-polyisoprene with Polyhedral Oligomeric Silsesquioxane-modified Side Chains (PS-b-PIPOSS)

The $\mathrm{PS}_{1200}-b$-PI 94 (0.2 g, $0.143 \mathrm{mmol} /$ vinyl groups), hydrido-heptacyclopentyl substituted POSS $(0.142 \mathrm{~g}$, $0.157 \mathrm{mmol})$ and toluene $(2 \mathrm{~mL})$ were added to a dry $20 \mathrm{~mL}$ Schlenk-type glasstube with a rubber septum cap and a stir bar under an argon atmosphere. After the PS- $b$-PI and the POSS dissolved, Karstedt's catalyst of platinum[0]-1,3-divinyl-1,1,3,3-tetramethyl disiloxane complex solution in xylene [Pt(dvs)] $(0.1$ $\left.\mathrm{mol}^{-1}\right)(7.2 \mu \mathrm{l}, 0.5 \mathrm{~mol} \%)$ was added via microsyringe to the glasstube at room temperature. Then, the mixture was stirred at $80^{\circ} \mathrm{C}$ for $24 \mathrm{~h}$. The solution was poured into methanol. The precipitate was collected, washed with methanol and dissolved in toluene, and then the solution was poured into methanol again. This precipitation was repeated three times to remove a small excess of the POSS and the catalysts. Finally, the precipitate collected was dried in vacuo at $50^{\circ} \mathrm{C}$ for $24 \mathrm{~h}$ to yield $0.289 \mathrm{~g}(88 \%)$ of PS- $b$-PIPOSS, $M_{\mathrm{n}}=$ 152,000 and PDI $=1.14 . \mathrm{IR}(\mathrm{NaCl}): v\left(\mathrm{~cm}^{-1}\right)=3059$, 3025, 2980, 2840 (C-H, st), 1600 (benzene, st), 1105 $(\mathrm{Si}-\mathrm{O}, \mathrm{st}) .{ }^{1} \mathrm{H}$ NMR $\left(300 \mathrm{MHz}, \mathrm{CDCl}_{3}\right) \delta(\mathrm{ppm})=$ 0.85-1.11 (br, Si-CH-cyclopentyl, $\mathrm{Si}-\mathrm{CH}_{2}-\mathrm{CH}_{2}$-polyisoprene), 1.20-2.28 (br, $\left.\mathrm{CH}_{2}, \mathrm{CH}\right), 4.63\left(\mathrm{br},-\mathrm{C}\left(\mathrm{CH}_{3}\right)=\right.$ $\mathrm{CH}_{2}$ ), 6.38-7.24 (br, benzene). ${ }^{13} \mathrm{C}$ NMR (75 Mhz, $\left.\mathrm{CDCl}_{3}\right) \delta(\mathrm{ppm})=13.5,21.8,22.1,25.4,25.6,26.9$, 27.0, 40.7, 40.9, 42.9, 44.2, 46.0, 125.7, 125.8, 127.5, 127.6, 127.9, 128.1, 128.4, 145.4, 145.9, 146.3.

\section{RESULTS AND DISCUSSION}

\section{Synthesis of Silicon-Containing Rod-Coil Type Block Copolymer}

The synthetic procedure of the polystyrene- $b$-polyisoprene with the POSS-modified side chains (PS- $b$ PIPOSS) is illustrated in Scheme 1. As the base polymer, polystyrene- $b$-poly(1,2-ran-3,4-isoprene) block copolymer having vinyl groups in the side chain (PS-b-PI), which is one of typical coil-coil type block copolymers, was prepared by anionic polymerization of styrene and isoprene with the number of repeat units of 1200 and 94, respectively. It is known that this coil-coil type block copolymer can be easily converted to a series of rod-coil type block copolymers by incorporation of a variety of rigid-rod molecules into the side chains. ${ }^{25-29}$ The synthesis of polystyrene- $b$ polyisoprene with the POSS-modified side chains (PS- $b$-PIPOSS) as a silicon-containing rod-coil type block copolymer was carried out by hydrosilation of polystyrene- $b$-poly(1,2-ran-3,4-isoprene) with the hydrido-heptacyclopentyl substituted POSS in the presence of a catalytic amount of $\operatorname{Pt}(\mathrm{dvs})$ in toluene at 


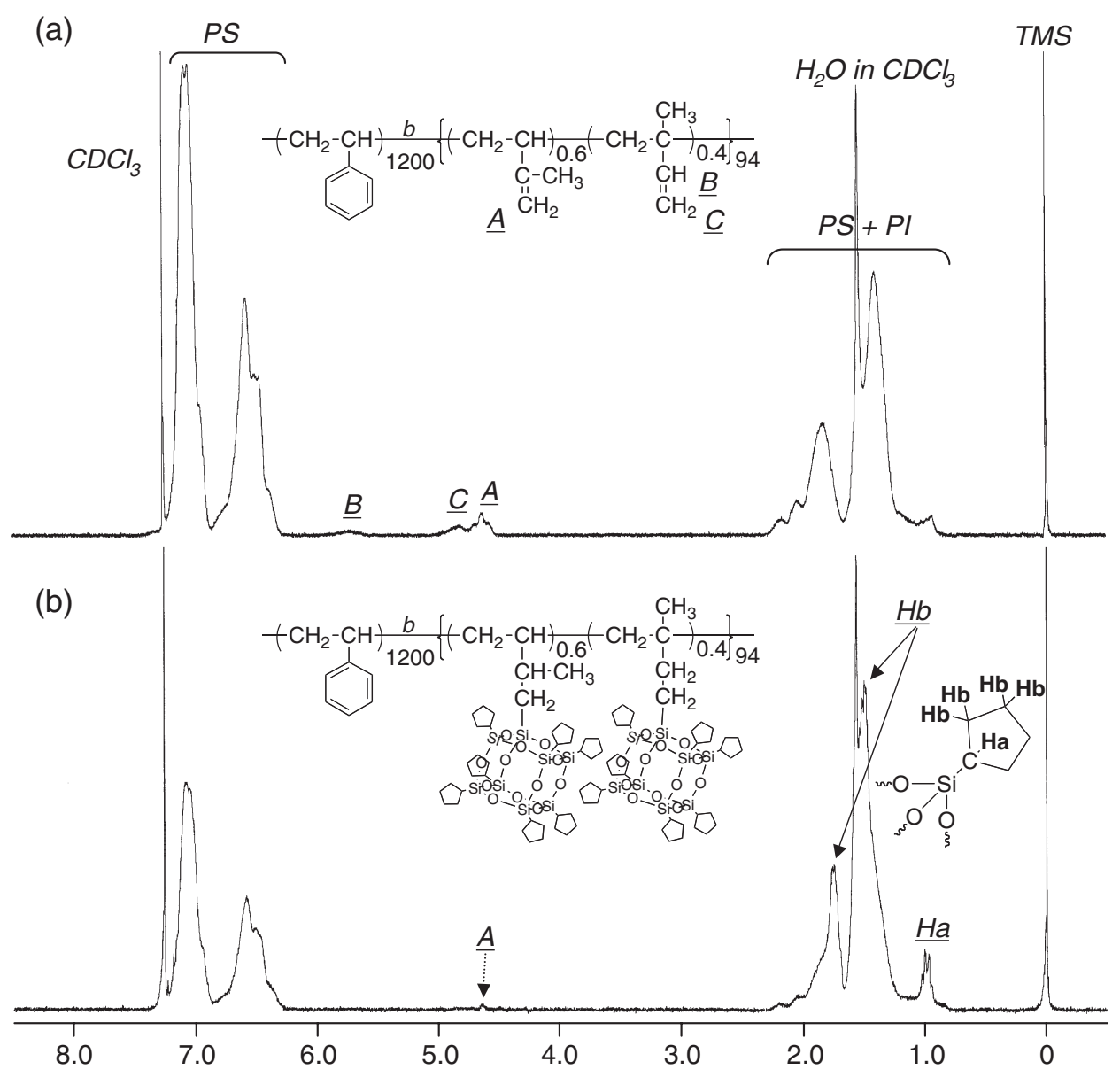

Figure 1. ${ }^{1} \mathrm{H}$ NMR spectra of (a) PS- $b$-PI and (b) PS- $b$-PIPOSS in $\mathrm{CDCl}_{3}$.

$80^{\circ} \mathrm{C}$ for $24 \mathrm{~h}$. The reaction was proceeded in homogeneously. After the reaction, the solution was poured into methanol. The precipitate was filtered, washed with methanol and dissolved in toluene, and then the solution was poured into methanol again. This purification repeated three times to remove a catalyst of $\mathrm{Pt}(\mathrm{dvs})$ and a small excess amount of the hydridoheptacyclopentyl substituted POSS. The yield of PS$b$-PIPOSS was $88 \%$.

The structure of the resulting PS- $b$-PIPOSS was characterized by IR and NMR spectroscopies. In the IR spectrum, peaks due to the Si-O stretching vibration at $1105 \mathrm{~cm}^{-1}$ corresponding to the siloxane bonds of the POSS were observed. The degree of hydrosilation of the POSS to the 1,2- and 3.4-isoprene units was calculated from ${ }^{1} \mathrm{H}$ NMR spectra. The peaks associated with the double bonds were used in determining the conversions of the 1,2- and 3.4-isoprene blocks. As shown in Figure 1, the two peaks at 4.86 $\left(-\mathrm{CH}=\mathrm{CH}_{2}\right)$ and $5.72\left(-\mathrm{CH}=\mathrm{CH}_{2}\right)$ ppm of PS- $b$-PI were completely disappeared in the ${ }^{1} \mathrm{H}$ NMR spectrum of the resulting PS- $b$-PIPOSS. However, very weak signal at $4.63 \mathrm{ppm}$ corresponding to the double bond of $-\mathrm{C}\left(\mathrm{CH}_{3}\right)=\mathrm{CH}_{2}$ was observed in the spectrum. This analysis of ${ }^{1} \mathrm{H}$ NMR spectrum reveals that $92 \%$ of the hydrido-heptacyclopentyl substituted POSS was incorporated into the block copolymer. Even with excess amount of the POSS, the catalyst, longer reaction times, higher conversion was not achieved. The proton signal associated with the hydridosilyl groups of the POSS at $4.12 \mathrm{ppm}$ was not observed in the resulting polymer. This indicates that the slightly excess amount of the POSS were able to be removed completely by the reprecipitation steps. The proton signals corresponding to the heptacyclopentyl groups of the PIPOSS block was observed around 1.2-1.8 ppm, but the precise assignment was difficult due to the overlap of the proton signals of the methylene units of PS- $b$-PI main chain. In the ${ }^{13} \mathrm{C}$ NMR spectrum, characteristic three signals were observed at 22.1, 25.4 and $27.0 \mathrm{ppm}$ due to the methane and methylene groups of the cyclopentyl groups, respectively. The other signals were also successfully assigned with the final product. Analysis of the NMR spectra confirm the desired structure of PS- $b$-PIPOSS and reveals that the POSS was incorporated into the coil-coil type PS- $b$-PI in high yield without any side reactions.

Gel permiation chromatography (GPC) indicated a narrow polydispersity index of 1.14 with a number average of molecular weight $M_{\mathrm{n}}$ of 152,000 (versus 
polystyrene standard in tetrahydrofuran). The PS- $b$ PIPOSS was colorless solid and soluble in tetrahydrofuran, chloroform, dichloromethane, and toluene but insoluble in hexane, methanol and acetone. The thermal behavior of the PS- $b$-PIPOSS was examined by thermogravimetric analysis (TGA) and differential scanning calorimeter (DSC). In the TGA curve, the weight loss started at $345^{\circ} \mathrm{C}$ and continued slowly. $10 \%$ of weight was lost around $408^{\circ} \mathrm{C}$ in nitrogen. In the $2^{\text {nd }}$ DSC curve, the transition was observed at $104.5^{\circ} \mathrm{C}$ due to the glass transition of the PS block. No evidence for the transition of the PIPOSS block was observed in the DSC curve.

\section{Preparation of Self-Organized PS-b-PIPOSS Film}

The film was prepared by casting the $0.2 \mathrm{wt} \%$ PS$b$-PIPOSS solution $(0.2 \mathrm{~mL})$ in toluene at room temperature $\left(25^{\circ} \mathrm{C}\right)$ under a moist air flow on a variety of substrates such as glass slides, silicon wafers, epoxy resins or polyimide films. The humidity and flow rate were kept at $85 \%$ and $3.01 \mathrm{~min}^{-1}$, respectively. Observation under a polarized optical microscopy with a high-speed camera showed that the water vapor derived from the moist air flow condensed into droplets in the solution owing to the latent heat of evaporation of the solvent. The droplets of water immediately grew after the moist air flow was started, and interference color appeared on the solution surface, indicating the formation of ordered structure of water droplets of an order of micron with a narrow size distribution. Further evaporation of the solvent and water solidified the ordered structure. The solvent and water completely evaporated within $60 \mathrm{~s}$, and the structured films formed over an area of $1-2 \mathrm{~cm}^{2}$. When other solvents such as chloroform, dichloromethane and carbon disulfide were used instead of toluene, the films were obtained as well, but the film preparation time depends on the boiling temperature of the solvents. It took around $30 \mathrm{~s}$ when these volatile solvents were used.

Characterization of Self-Organized Hierarchically Structure and Chemically Heterogeneous Surface

Figure 2 shows the scanning electron microscopy (SEM) images which were obtained from the film prepared on a silicon wafer. Observation using SEM confirmed the formation of hexagonally packed micropores with a narrow size distribution. The diameter of the open pores was approximately $1.7 \mu \mathrm{m}$. A crosssectional view shows a single layer of empty spheres, which have a diameter of $2.2 \mu \mathrm{m}$ and are separated by the thin walls of less than approximately $100 \mathrm{~nm}$. AFM studies showed very smooth and flat surface morphology of the film. The surface roughness was less than $1 \mathrm{~nm}$ (Figure 3 ). (a)

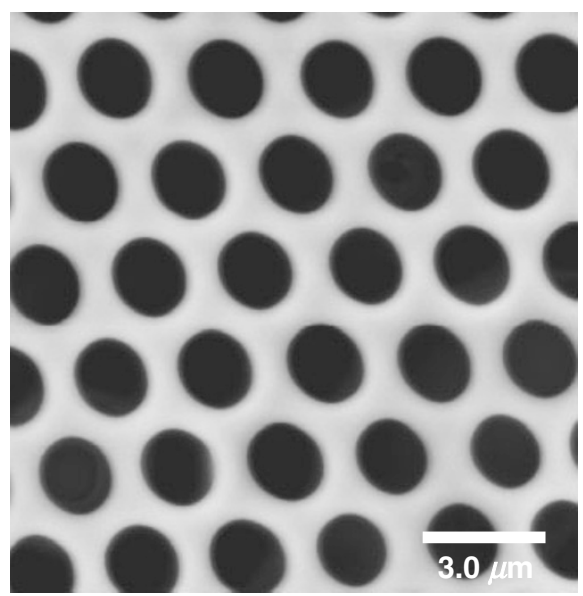

(b)

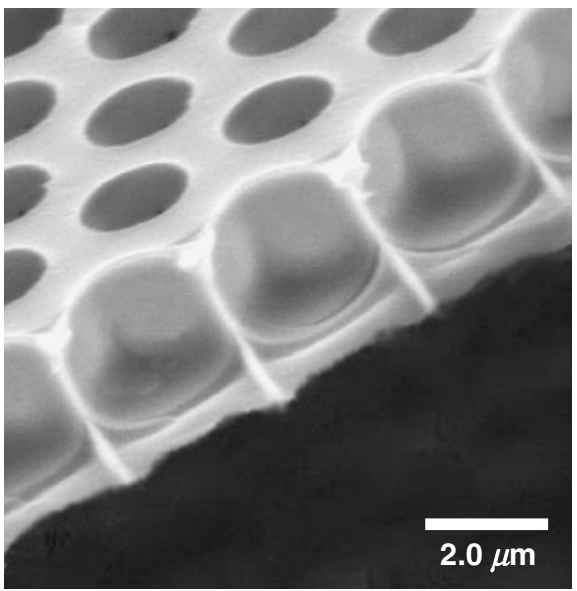

Figure 2. SEM images of self-organized PS- $b$-PIPOSS film with microporous structure. (a) Top view and (b) Side view of the perpendicularly cross-sectioned film.
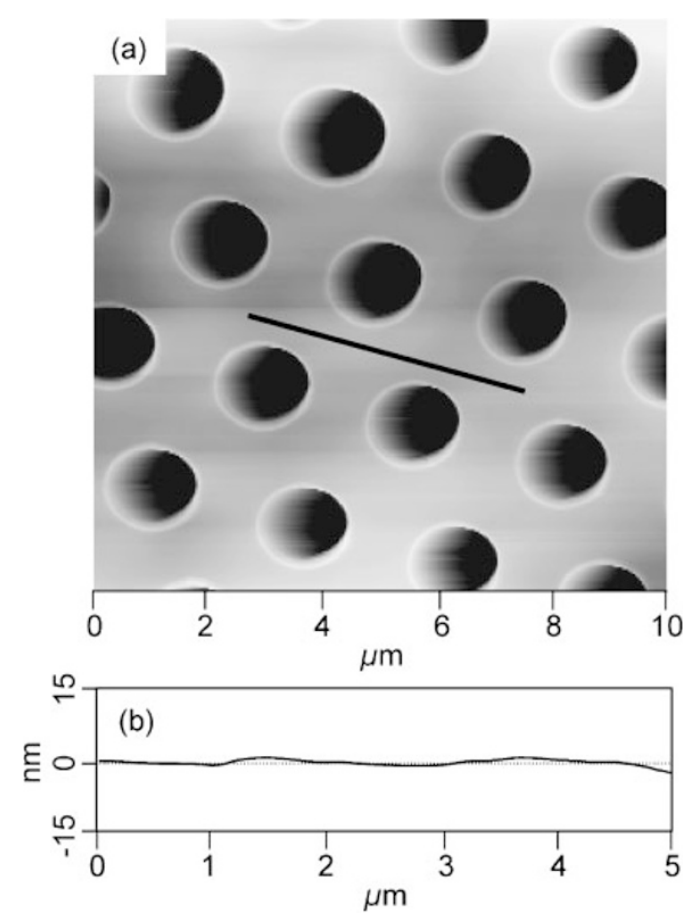

Figure 3. AFM image of self-organized PS- $b$-PIPOSS film with microporous structure. (a) Top view and (b) Cross-sectional profile along the line in image (a). 

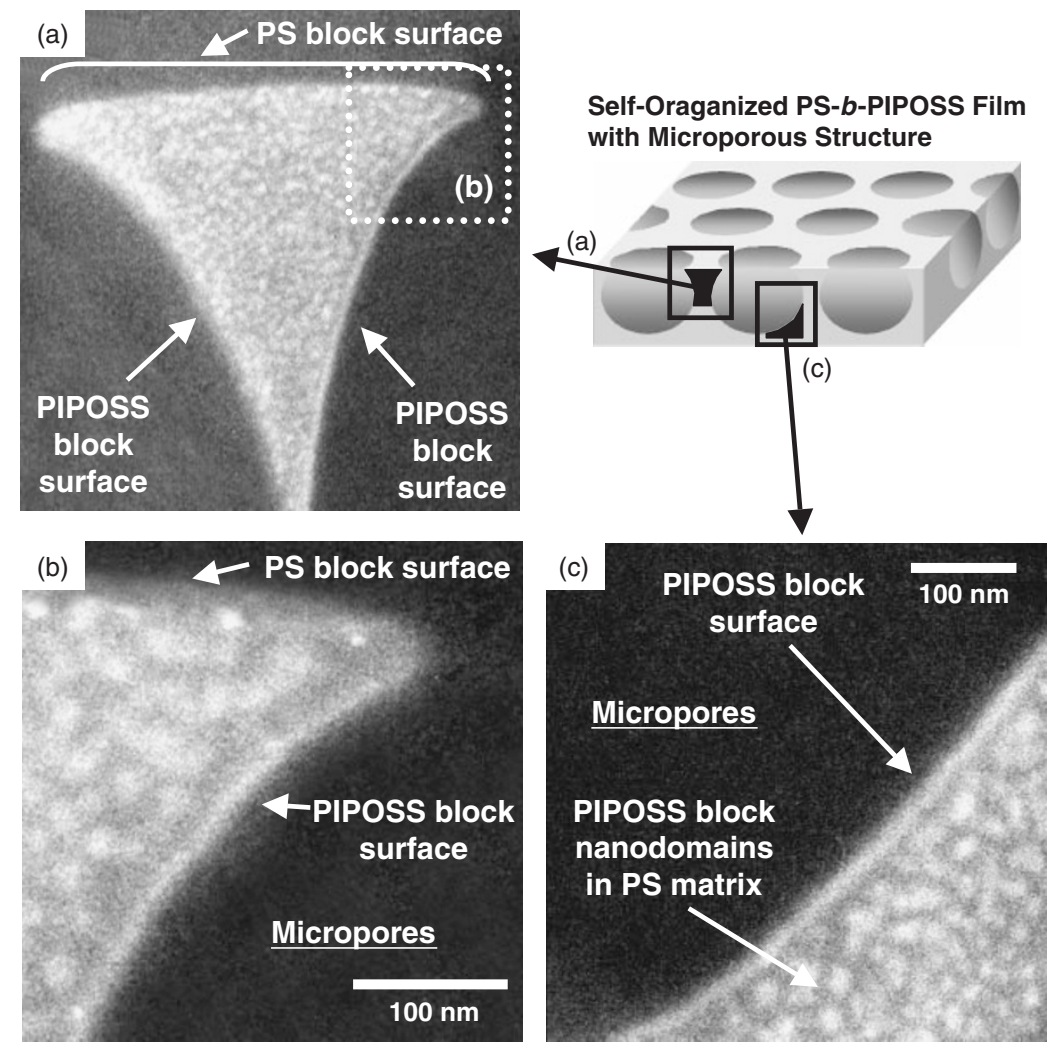

Figure 4. EFTEM images created with inelastically scattered electrons of $250 \pm 10 \mathrm{eV}$ energy losses of self-organized PS- $b$-PIPOSS film. The bright and dark color regions are corresponding to the PIPOSS and the PS nanodomains, respectively. (a) Perpendicularly crosssectioned microporous film (Medium-magnification). (b) High-magnification micrograph at the top-surface region of (a). The flat and curve areas indicate the surfaces are covered with the PS and the PIPOSS blocks, respectively. (c) High-magnification micrograph at the interiors of the microporous film.

In order to observe nanostructures of PS- $b$-PIPOSS in the film by using an energy-filtering transmission electron microscope (EFTEM), the film prepared on the epoxy plates with an area of $\sim 1 \mathrm{~cm}^{2}$ was cross-sectioned perpendicular to the substrate to obtain thin specimens with $50 \mathrm{~nm}$ thicnkness after embedding in epoxy resin. The micrographs in Figure 4 are the energy-filtered images with an inelastically-scattered electrons with $250 \pm 10 \mathrm{eV}$ energy losses. The EFTEM images clearly showed the hierarchically structures consist of the nanodomains within the micrometer-sized porous structures. The nanostructure was hardly observed in the corresponding conventional TEM image but clearly observed in the energyfiltered image at the energy loss level of $285 \mathrm{eV}$, which corresponds to the carbon K-edge. In a specimen where carbon is the majority element, optimum contrast is usually achieved at around $250 \mathrm{eV}$, where the domains containing less carbon appear bright. Therefore, the brighter nanodomains were corresponding to the PIPOSS block within the PS matrix, in which an averaged size of the nanodomains and an averaged distance between the nanodomains are approximately 25 and $35 \mathrm{~nm}$, respectively.

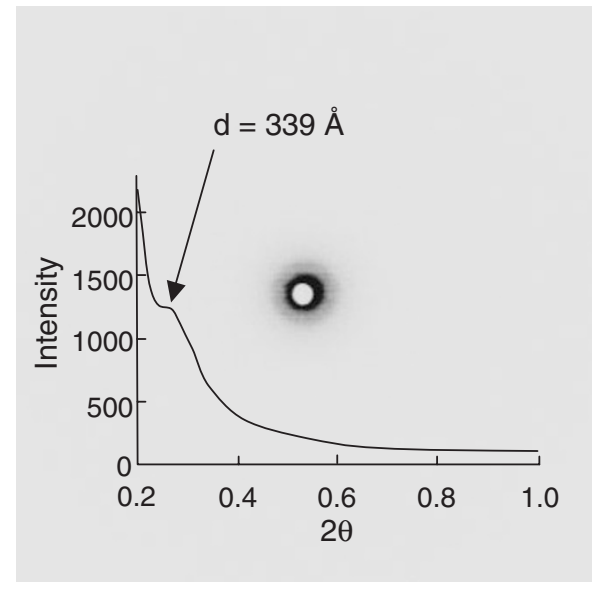

Figure 5. SAXS pattern and line profile of the self-organized PS- $b$-PIPOSS film.

A line scan of SAXS data for PS- $b$-PIPOSS is shown in Figure 5. The profile exhibits a domain spacing of $339 \AA$. This is a good agreement with the averaged distance between the nanodomains in EFTEM observation. These findings indicate that the PS- $b$-PIPOSS film is constructed by the hierarchically ordered structure which has three tiers of the micro- 


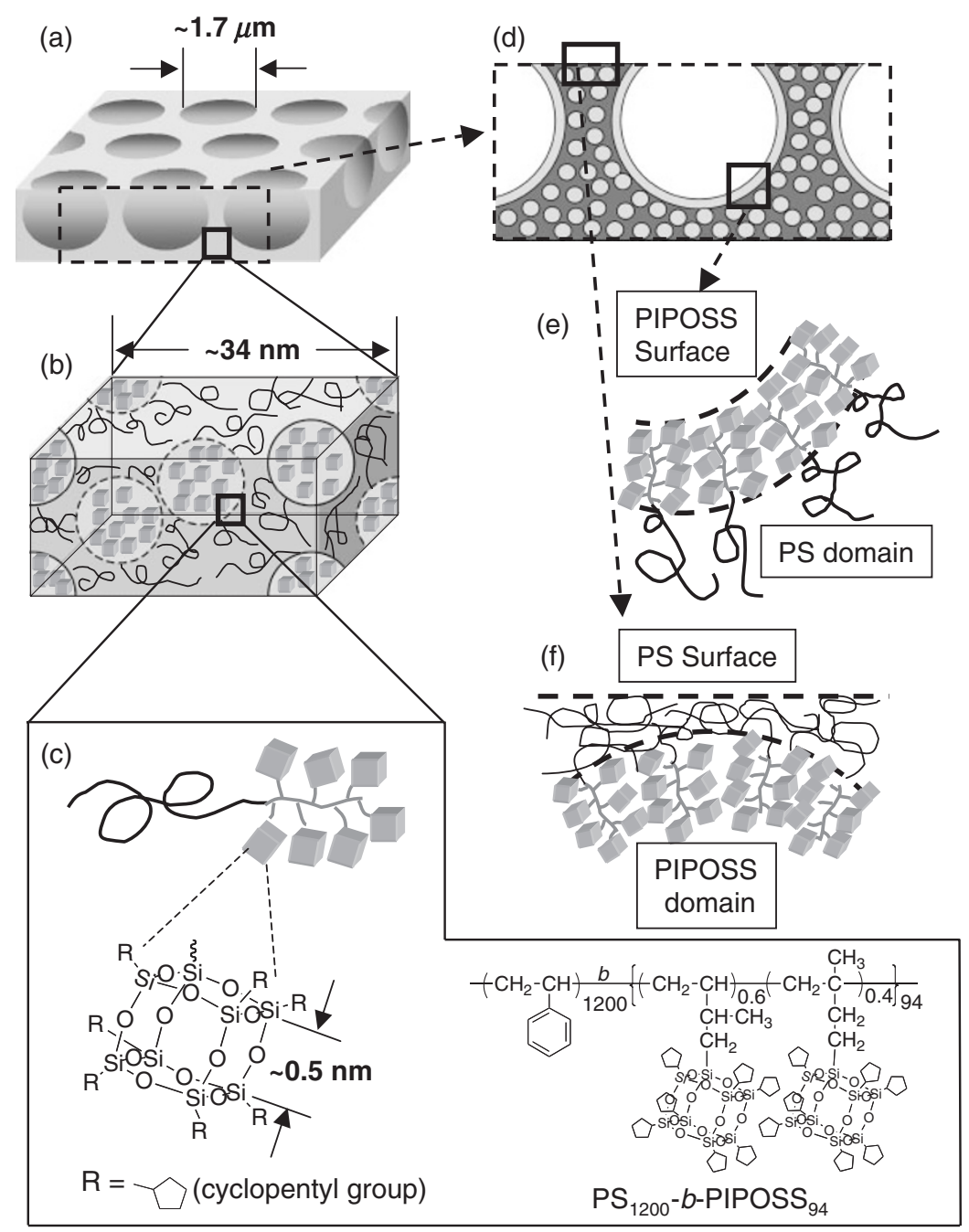

Figure 6. Schematic representation of self-organized hierarchically structure and chemically heterogeneous surface of PS- $b$-PIPOSS film. (a) microporous structure (b) phase-separated nanodomains (c) molecular structure of the PS- $b$-PIPOSS (d) cross-sectioned structure (e) self-organized structure at interior surface of the micropores (f) self-organized structure at flat surface of the film.

pores of which walls consist of the nanodomains of the PIPOSS containing the molecular cubic of POSS: the structure of the length scales from angstrom to micrometer (Figures 6a-6c).

Furthermore, it was also found the formation of the chemically heterogeneous surface in the resulting film. As reported in our recent study, ${ }^{22}$ the microporous film prepared from a solution of rod-coil type block copolymer under a moist air flow can form the chemically heterogeneous surface which the interiors of the pores and the flat areas on the surface were covered with the rod block and the coil block, respectively. As shown in Figure 4c, a bright color line was observed at the interiors of the pore. This indicates that the interiors of the pore on the surface are covered with the PIPOSS block. The thickness at the top layer is less than $\sim 20 \mathrm{~nm}$, based on TEM observations. Otherwise, there is no bright color region at the flat surface of the topologically microporous film (Figure 4b), which indicates the flat surface is covered with the PS block. The reason for the formation of the chemically heterogeneous surface in the microporous film is in the behavior of the rod-coil type block copolymer during the self-assembly of water droplets. In the self-assembling process of water droplets, the rod-coil type block copolymer plays a role as a surfactant to stabilize the inverse emulsion of water. In this case, the rod segment block of the PIPOSS block, which is relatively more hydrophilic than the PS block, is partitioned to the water side of the interface between the water droplets and the polymer solution. After evaporation of the solvent and water, the rod segment of the PIPOSS block remains at the interiors of the micropores. At the other surface, which had been the interface with air, the PS block is segregated due to the low surface energy component in the block copolymer. As a result, the chemically heterogeneous surface of the PS and the PIPOSS components are formed in the microporous film (Figures 6d-6f). 

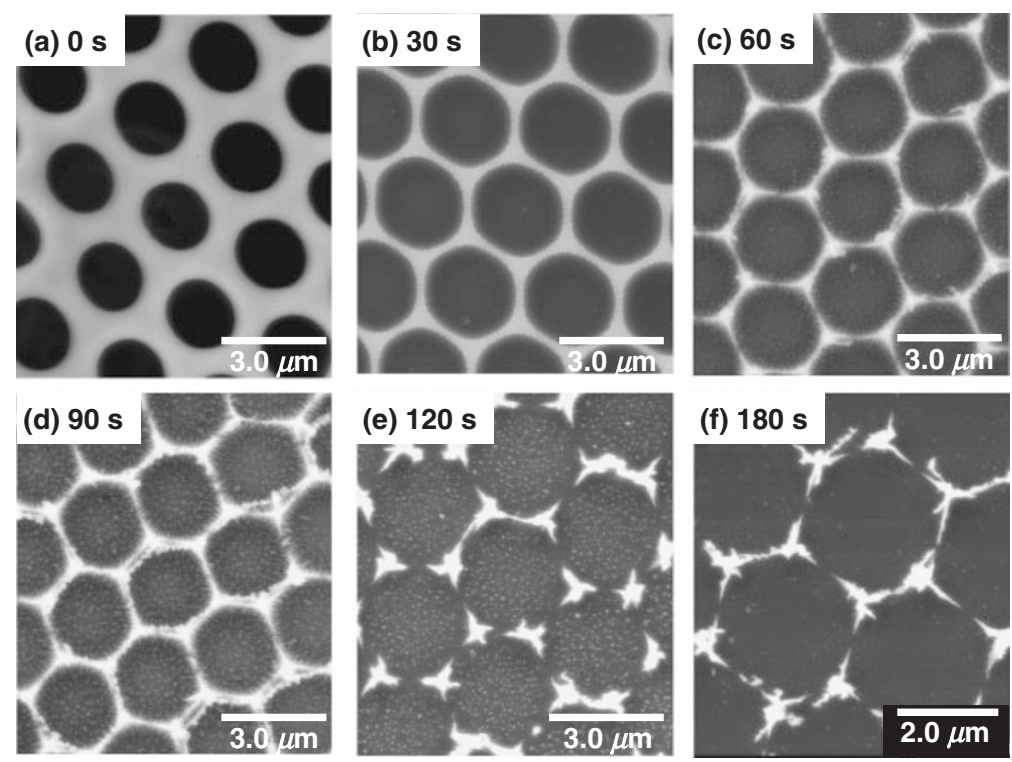

Figure 7. SEM images of the self-organized PS- $b$-PIPOSS film with microporous structure before etching and after 30 to $180 \mathrm{~s}$ of etching under oxygen plasma.
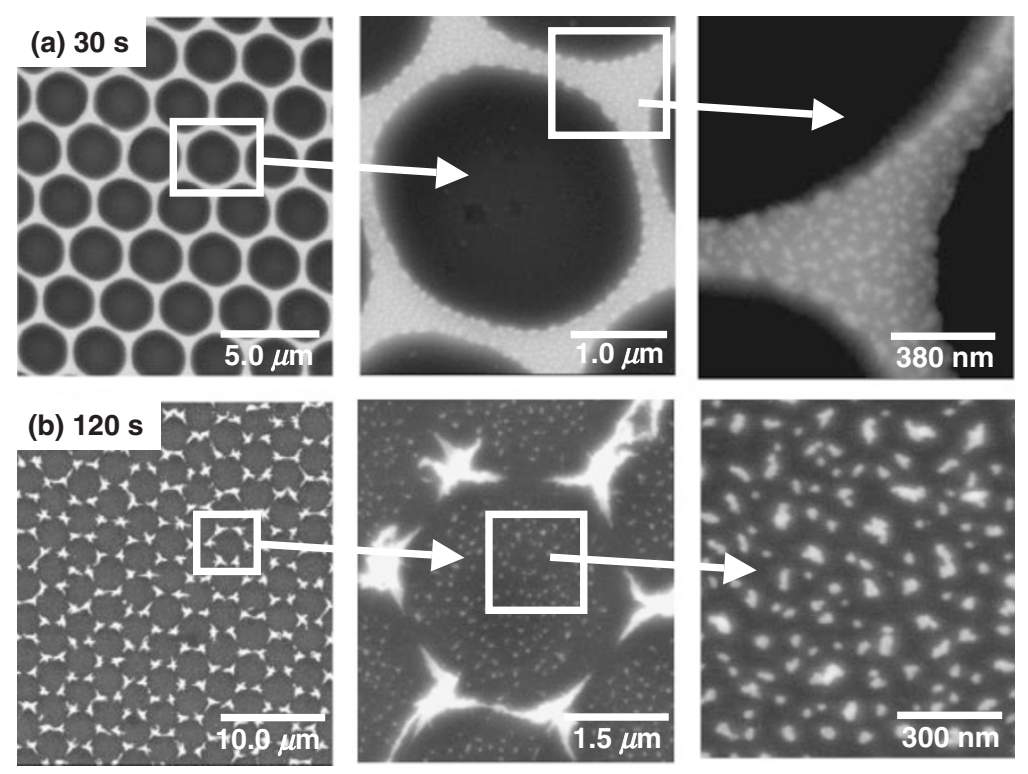

Figure 8. High-magnification SEM images of the self-organized PS- $b$-PIPOSS film with microporous structure after 30 and $120 \mathrm{~s}$ of etching under oxygen plasma.

Fabrication of Ordered Hierarchically Surface Structures by Applying Oxygen Plasma

Based on the hierarchically structure at length scales from nanometers to micrometers and the chemically heterogeneous surface of the self-organized PS- $b$-PIPOSS film, we attempt to fabricate the hierarchically ordered organic and inorganic hybrid structures under oxygen plasma. The etch resistance of the PIPOSS block to oxygen plasma may be higher than that of the PS block because of containing the silicon units of the POSS in the PIPOSS block. The difference of the etch resistance leads to selective etching of the chemically heterogeneous surface of the PS- $b$ -

\section{PIPOSS film.}

The films etched by oxygen plasma as a sequence of time are shown in Figure 7. When the etching time of the self-organized PS- $b$-PIPOSS film was $30 \mathrm{~s}$, the flat surface was selectively etched that had been covered with the PS block, and the dot-like nanostructures appeared in the SEM image (Figure 7b). The nanodots are obviously derived from the nanodomains of the PIPOSS block that were oxidized to the silicon oxide. The diameter of the open micropores on the surface increased to approximately $2.7 \mu \mathrm{m}$. Interestingly, the interiors of the micropores on the surface were not etched in this condition. With increasing 


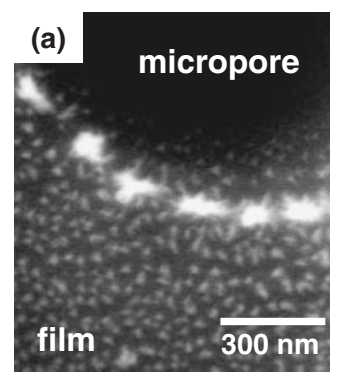

Figure 9. SEM images of the edge of the microporous film after $45 \mathrm{~s}$ of etching under oxygen plasma.

the etching time from 30 to $45,60,90$, and $120 \mathrm{~s}$, the interiors of the pores were gradually etched and simultaneously the nano-dots appeared (Figures 7b-7f). After the oxygen plasma treatment of $180 \mathrm{~s}$, the nanodots almost disappeared in the film, but the hexagonally packed array structure still remained. The diameter of the nano-dots are less than $\sim 20 \mathrm{~nm}$, based on SEM observations. To compare with the films which were etched with 30 and $120 \mathrm{~s}$, it clearly can be seen that two different types of the hierarchically surface structures were obtained by the fabrication of the original self-organized films via the simple oxygen plasma treatment (Figure 8).

As shown in Figure 9, the SEM images of the etched film with $45 \mathrm{~s}$ indicate the remaining domains of the PIPOSS block at the edge of the microporous film. This fact evidently indicates that the interiors of the micropores had been covered with the PIPOSS block. This caused by the chemically heterogeneous surface and silicon-containing hierarchically structures of the PS- $b$-PIPOSS film (Figure 10).

\section{CONCLUSIONS}

We have demonstrated a facile fabrication of hierarchically ordered organic and inorganic hybrid structures based on the self-organized hierarchically structures of the POSS functionalized rod-coil type block copolymer film and an oxygen plasma treatment. The rod-coil type block copolymer, PS- $b$-PIPOSS, was successfully prepared by incorporation of rigid-rod silicon molecule of the POSS into the coil-coil type block copolymer of polystyrene- $b$-poly $(1,2-$ ran-3,4isoprene) via hydrosilation. The hexagonally ordered microporous film of the PS- $b$-PIPOSS was obtained by casting of the solution in toluene onto silicon wafers under a moist air flow. The PIPOSS block was self-assembled into the PS matrix and formed nanometer-scale domains with a size of approximately $25 \mathrm{~nm}$. It was observed the formation of the chemically heterogeneous surface consisting of the PS and the PIPOSS components in the film, in which the interiors of the micropores on the surface were covered with

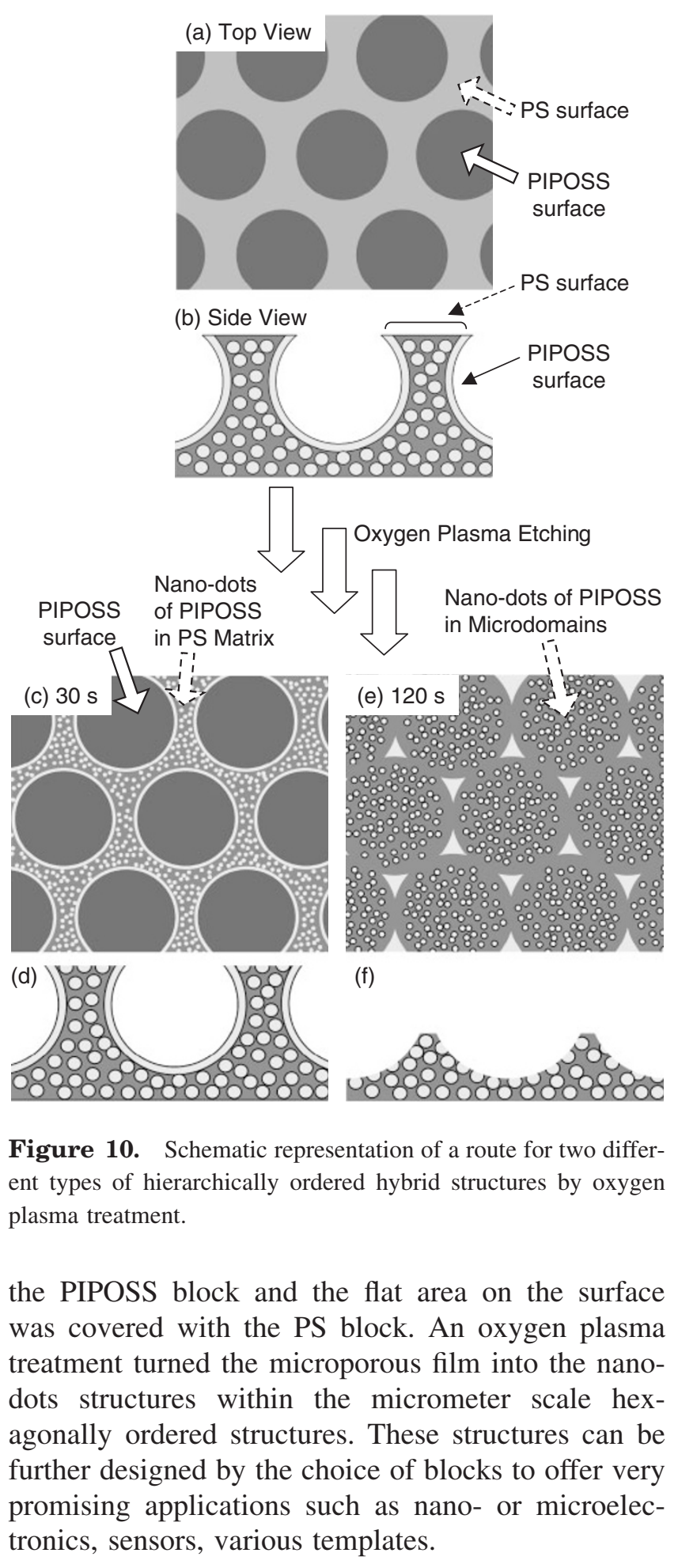

\section{REFERENCES}

1. J. M. Weissman, H. B. Sunkara, A. S. Tse, and S. A. Asher, Science, 274, 959 (1996).

2. E. A. Kamenetzky, L. G. Magliocco, and H. P. Panzer, Science, 263, 207 (1994).

3. S. H. Sun, C. B. Murray, D. Weller, L. Folks, and A. Moser, Science, 287, 1989 (2000).

4. B. J. Siwick, O. Kalinina, E. Kumacheva, R. J. D. Miller, and J. Noolandi, J. Appl. Phys., 90, 5328 (2001).

5. H. H. Pham, I. Gourevich, J. K. Oh, J. E. N. Jonkman, and 
E. Kumacheva, Adv. Mater., 16, 516 (2004).

6. J. H. Holtz and S. A. Asher, Nature, 389, 829 (1997).

7. O. D. Velev and E. W. Kaler, Langmuir, 15, 3693 (1999).

8. S. A. Asher, V. L. Alexeev, A. V. Goponenko, A. C. Sharma, I. K. Lednev, C. S. Wilcox, and D. N. Finegold, J. Am. Chem. Soc., 125, 3322 (2003).

9. A. C. Sharma, T. Jana, R. Kesavamoorthy, L. J. Shi, M. A. Virji, D. N. Finegold, and S. A. Asher, J. Am. Chem. Soc., 126, 2971 (2004).

10. G. M. Wallraff and W. D. Hinsberg, Chem. Rev., 99, 1801 (1999).

11. K. P. Gritsenko and A. M. Krasovsky, Chem. Rev., 103, 3607 (2003).

12. Y. Xia and G. M. Whitesides, Angew. Chem., Int. Ed., 37, 551 (1998).

13. M. Li, K. Douki, K. Goto, X. Li, C. Coenjarts, D. M. Smilgies, and C. K. Ober, Chem. Mater., 16, 3800 (2004).

14. P. Jiang and M. J. McFarland, J. Am. Chem. Soc., 126, 13778 (2004).

15. F. S. Bates and G. H. Fredrickson, Annu. Rev. Phys. Chem., 41, 525 (1990).

16. C. Park, J. Yoon, and E. L. Thomas, Polymer, 44, 6725 (2003).

17. A. H. Gobor, E. A. Lehner, G. Mao, L. A. Schneggenburger, and C. K. Ober, Chem. Mater., 6, 927 (1994).

18. R. G. H. Lammertink, M. A. Hempenius, V. Z.-H. Chan,
E. L. Thomas, and G. J. Vancso, Chem. Mater., 13, 429 (2001).

19. K. Fukukawa, L. Zhu, P. Gopalan, M. Ueda, and S. Yang, Macromolecules, 38, 263 (2005).

20. M. Lee, B.-K. Cho, and W.-C. Zin, Chem. Rev., 101, 3869 (2001).

21. T. Hayakawa and S. Horiuchi, Angew. Chem., Int. Ed., 42, 2285 (2003).

22. T. Hayakawa and H. Yokoyama, Langmuir, 21, 10288 (2005).

23. A. J. Waddon and E. B. Coughlin, Chem. Mater., 15, 4555 (2003).

24. Y. Abe and T. Gunji, Prog. Polym. Sci., 29, 149 (2004).

25. G. Mao, J. Wang, S. R. Clingman, and C. K. Ober, Macromolecules, 30, 2556 (1997).

26. J. Wang, G. Mao, C. K. Ober, and E. J. Kramer, Macromolecules, 30, 1906 (1997).

27. T. Hayakawa, J. Wang, M. Xiang, X. Li, M. Ueda, C. K. Ober, J. Genzer, E. Sivaniah, E. J. Kramer, and D. A. Fisher, Macromolecules, 33, 8012 (2000).

28. T. Hayakawa, J. Wang, N. Sundararajan, M. Xiang, X. Li, B. Glüsen, G. C. Leung, M. Ueda, and C. K. Ober, J. Phys. Org. Chem., 13, 787 (2000).

29. E. Sivaniah, J. Genzer, G. H. Fredrickson, E. J. Kramer, M. Xiang, X. Li, C. Ober, and S. Magonov, Langmuir, 17, 4342 (2001). 